\title{
The Antarctic Oscillation Structure in an AOGCM with Interactive Stratospheric Ozone
}

\author{
S. Brand, K. Dethloff, and D. Handorf \\ Foundation Alfred Wegener Institute for Polar and Marine Research, Research Unit Potsdam, Telegrafenberg A45, \\ 14473 Potsdam, Germany
}

Correspondence should be addressed to S. Brand, sascha.brand@awi.de

Received 5 March 2012; Accepted 7 May 2012

Academic Editors: I. Bordi and N. Wang

Copyright (๑) 2012 S. Brand et al. This is an open access article distributed under the Creative Commons Attribution License, which permits unrestricted use, distribution, and reproduction in any medium, provided the original work is properly cited.

Based on 150-year equilibrium simulations using the atmosphere-ocean-sea ice general circulation model (AOGCM) ECHOGiSP, the southern hemisphere winter circulation is examined focusing on tropo-stratosphere coupling and wave dynamics. The model covers the troposphere and strato-mesosphere up to $80 \mathrm{~km}$ height and includes an interactive stratospheric chemistry. Compared to the reference simulation without interactive chemistry, the interactive simulation shows a weaker polar vortex in the middle atmosphere and is shifted towards the negative phase of the Antarctic Oscillation (AAO) in the troposphere. Differing from the northern hemisphere winter situation, the tropospheric planetary wave activity is weakened. A detailed analysis shows, that the modelled AAO zonal mean signal behaves antisymmetrically between troposphere and strato-mesosphere. This conclusion is supported by reanalysis data and a discussion of planetary wave dynamics in terms of Eliassen-Palm fluxes. Thereby, the tropospheric planetary wave activity appears to be controlled from the middle atmosphere.

\section{Introduction}

Large-scale atmospheric variability modes as Arctic Oscillation/Northern Annular Mode (AO/NAM) for the northern hemisphere (NH) and Antarctic Oscillation/Southern Annular Mode (AAO/SAM) for the southern hemisphere $(\mathrm{SH})$ are widely accepted to play an important role in the extratropical circulation (see, e.g., Thompson and Wallace [1]). However, when discussing these variability modes associated with tropo-stratospheric dynamical coupling and atmospheric wave phenomena, it is often focused on one hemisphere. Physical or chemical mechanisms are presumed to be similar for both hemispheres, and differences of circulation and processes are directly attributed to different lateral and lower boundary conditions due to land-sea distribution and topography. Although this attribution is undoubted, there can be important internal shifts within the climate system. For example, considering that the strato-mesospheric polar vortex is much stronger for the $\mathrm{SH}$ than for the $\mathrm{NH}$ winter, the tropo-stratosphere coupling by gravity waves (see Holton [2]) and planetary waves (see Charney and Drazin [3]) can be substantially effected. Thus, under differing atmospheric conditions, the relative importance of interacting process chains within the climate system might significantly change.

Model intercomparisons, as done, for example, by Miller et al. [4] using 21st century projections of the Fourth Assessment Report IPCC models, show a wide spread of the results not only between the models, but also in particular between the $\mathrm{NH}$ and $\mathrm{SH}$. Although generally, especially for the SH mainly as a consequence of greenhouse gas increase and stratospheric ozone depletion (see Shindell and Schmidt [5]), an increase of the NAM/SAM index is projected, this trend might even reverse in the $\mathrm{SH}$ summer due to stratospheric ozone recovery (see Perlwitz et al. [6] and Son et al. [7]). Furthermore, for example, Scaife et al. [8] demonstrated that the positive trend of the North Atlantic Oscillation (NAO) index in the $\mathrm{NH}$ tropospheric circulation, associated to a trend of the AO, is typically modelled too weakly and can only be fully simulated by imposing observed trends in the lower stratosphere. Thus, at least some of the intermodel discrepancies within the IPCC projections are likely to be a result of unrealistic stratospheric 
model representations with prescribed ozone forcing in some models.

A global model setup as the AOGCM ECHO-GiSP with fully interactive stratospheric chemistry (see the following section) can help to address such issues. Ozone chemistry is most important in the stratosphere but also plays a major role regarding the impact of atmospheric chemistry on radiation and thus on atmospheric dynamics in general. This already implies the need for a model version, which covers not only the troposphere, but also the stratosphere. Additionally, one has to be aware that a low model top could lead to problems with the downward control mechanism (see Haynes et al. [9]) at polar latitudes.

Furthermore, global simulations enable the evaluation and comparison of the data for both hemispheres. For the $\mathrm{NH}$ winter, key results of a reference simulation (REF) and a simulation with enabled interactive chemistry-dynamics coupling (COUP) are shown in Brand et al. [10]. Comparable results for the $\mathrm{SH}$ winter, including zonal means and a discrete Fourier analysis, extended by an analysis of EliassenPalm fluxes, are presented here.

\section{Technical Remarks}

2.1. Model. ECHO-GiSP ("ECHO-G with integrated stratospheric chemistry by AWI Research Unit Potsdam") is an extension of the coupled AOGCM ECHO-G ("ECHAM and HOPE-G" see Legutke and Voss [11]). A chemistry part based on the MECCA chemistry module ("Module Efficiently Calculating the Chemistry of the Atmosphere" see Sander et al. [12]) was implemented. The model includes the 39-level middle atmosphere version (see Manzini et al. [13] and Manzini and McFarlane [14]) of the atmosphere model ECHAM4 (see Roeckner et al. [15]) with a model top at $1 \mathrm{~Pa}(80 \mathrm{~km})$. It was applied at a spectral resolution of T30, which relates to a rather coarse grid but is enough to capture dynamic effects due to the introduced stratospheric chemistry.

As ocean component, the ocean-sea ice model HOPE-G (see Wolff et al. [16]) using a horizontal resolution of T42 with a refinement towards the equator and a vertical resolution of 21 levels is included in ECHO-G. Thereby, a flux correction is applied for heat and freshwater exchange between atmosphere and ocean.

The chemistry scheme, which was integrated into the ECHAM part of ECHO-GiSP, allows gas-phase reactions, photolysis reactions, and heterogeneous reactions on polar stratospheric clouds. We used a configuration of the stratospheric chemistry with 116 chemical reactions (81 gasphase, 25 photolysis and 10 heterogeneous reactions) and 39 chemical species to perform the model simulations discussed in this paper. Beside the main members of the $\mathrm{O}_{x}, \mathrm{NO}_{x}$, $\mathrm{ClO}_{x}, \mathrm{HO}_{x}$, and $\mathrm{BrO}_{x}$ chemical families, species as $\mathrm{CO}$, $\mathrm{CO}_{2}, \mathrm{CH}_{4}, \mathrm{~N}_{2}, \mathrm{H}_{2}$, and $\mathrm{H}_{2} \mathrm{O}$ are included. All species are transported via the semi-Lagrangian transport scheme of Rasch and Williamson [17].

The interactive feedback between chemistry and dynamics can be enabled by using the simulated trace gas concentration of ozone $/ \mathrm{O}_{3}$ (Figure $1(\mathrm{a})$ ) within the $\mathrm{ECHO}-\mathrm{G}$ radiation scheme (see Morcrette [18]) instead of the predefined climatological ozone parameterisation (Figure 1(b)). If the interactive chemistry-dynamics mode is disabled, ECHOGiSP is dynamically equivalent to the EGMAM model of the institute for meteorology of Freie Universität Berlin (IfMFUB), which is validated, for example, in Huebener et al. [19].

Water is present in the chemistry scheme but can even in the interactive model version not feed back to the radiation scheme. This means that the information about the $\mathrm{H}_{2} \mathrm{O}$ mixing ratio from ECHO-G is taken into account within MECCA but is not recalculated and given back to the atmosphere model. Thus, the differences in the ozone fields used within the radiation scheme of the atmosphere model (Figure 1(c)) are the only reason for differing results when running the model with or without interactive stratospheric chemistry. Particularly, the simulations performed for this study use present day forcing conditions, and there are no external trends of the solar constant or trace gas concentrations.

Since our main focus was on impacts of stratospheric chemistry, data from the KASIMA chemistry transport model ("Karlsruhe simulation model of the middle atmosphere" see Kouker et al. [20]) have been used to provide boundary conditions for the chemical fields in the troposphere and the initial conditions on the entire grid. The use of this model setup allows interactive chemistry and dynamical feedbacks in the stratosphere, while keeping prescribed chemistry in the troposphere, avoiding a too complex chemistry scheme. There is no explicit prescription of surface sources and sinks for the chemical species used, which would introduce additional uncertainties. However, the modelled ozone concentration with enabled interactive stratospheric chemistry shows a good agreement with ozone measurements from satellite data (Figure 1(d)).

2.2. Simulations. An important advantage of the simplified stratospheric chemistry within ECHO-GiSP is that this enabled us to perform two fully coupled atmosphere-ocean long-term simulations of 150 simulation years each. Both of them are time-slice experiments ran under fixed present-day conditions, starting from the same initial state and differing only in the trace gas concentrations used in the ECHO-G radiation scheme.

For the reference run, hereafter denoted as REF, the climatological trace gas fields were used. The dynamical variables were not influenced by the modelled chemical constituents, causing the equivalence with the results from the EGMAM model of IfM-FUB. The validation for this model by Huebener et al. [19] concluded that the presentday climate is realistically simulated.

On the other hand, the so-called coupled run (COUP) was performed with the interactive chemistry-dynamics coupling switched on, that is, the radiation scheme was driven by the simulated ozone field in the stratosphere. Figure 2 shows the zonal mean temperature and zonal mean zonal wind for both winter periods (JJA at SH, DJF at NH) in this simulation, which appear reasonable. 


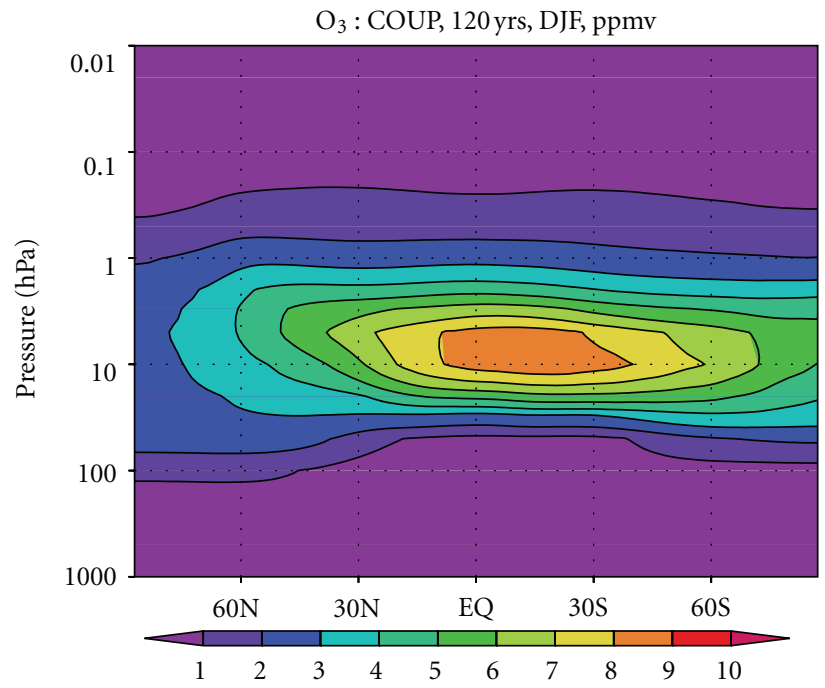

(a)

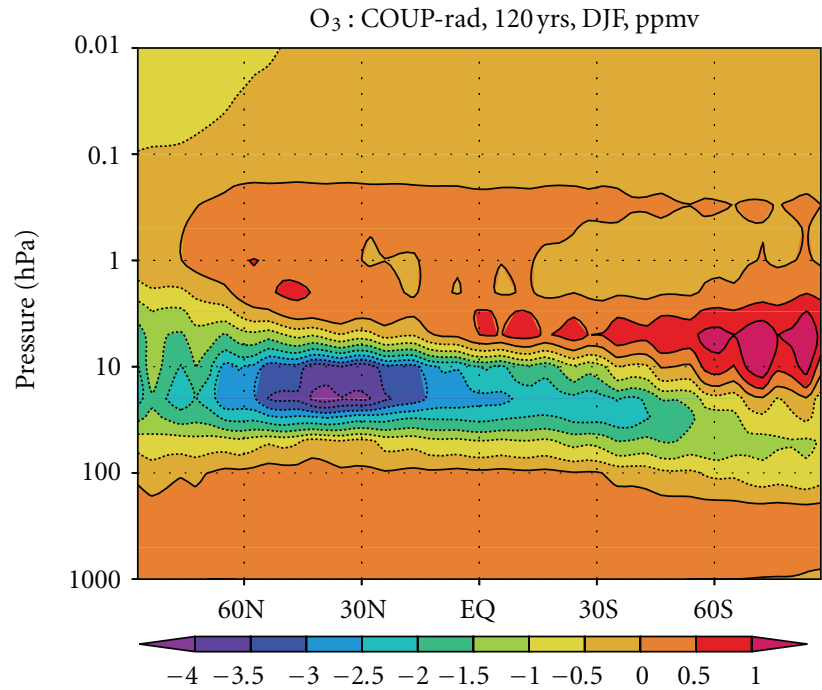

(c)

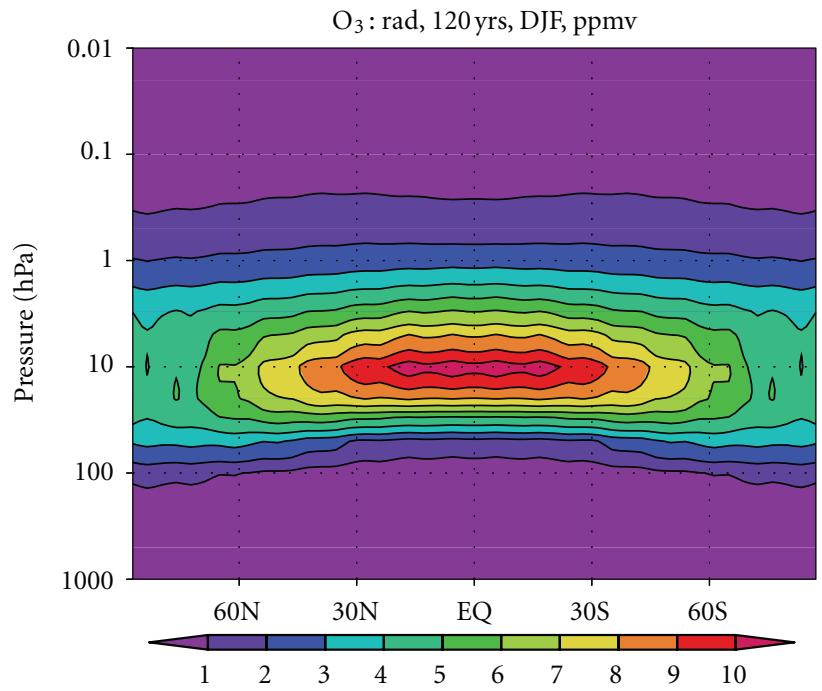

(b)

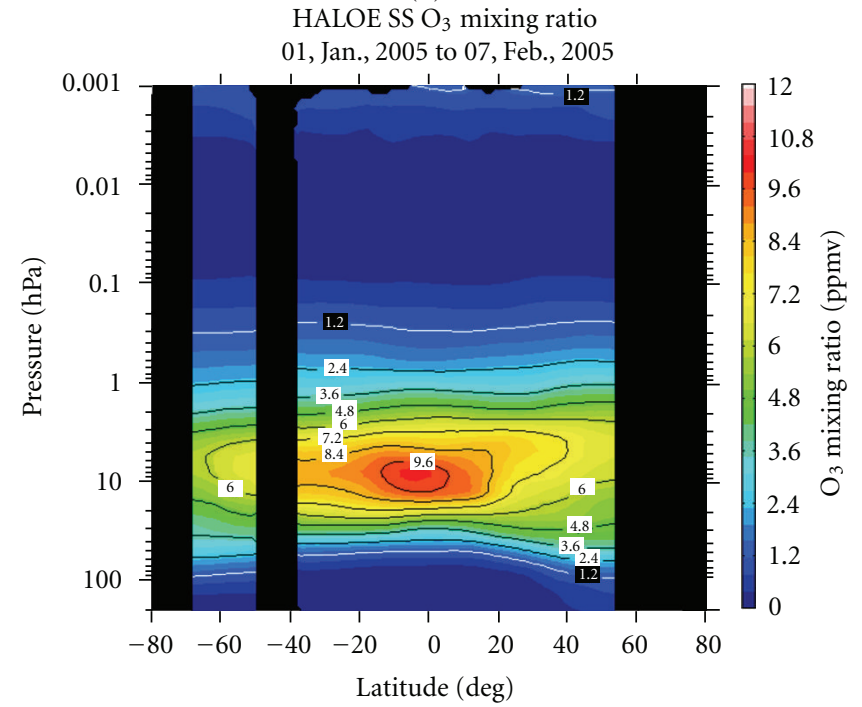

(d)

FIGURE 1: Ozone: zonal mean ozone concentration in (ppmv). Simulation years from 31 to 150. Example for NH winter (DJF): (a) interactive ozone within COUP; (b) parameterized ozone within REF; (c) differences between interactive and parameterized ozone; (d) zonal mean ozone concentration from HALOE satellite measurements for 01.01.-07.02.2005 (sunset) in (ppmv). Note that north and south are swapped, latitudes are cut down, and the vertical resolution differs compared to (a)-(c).

For both of our simulations (COUP and REF), we chose a spin-up time of 30 years. Thereby, the relatively long equilibration period of 30 years was chosen due to the coupling with an interactive ocean. This generally requires a longer adjustment phase than only for the atmospherechemistry system.

2.3. Methods. As a convention, the geopotential height field of the $x \mathrm{hPa}$ pressure surface is denoted as $\mathrm{z}_{x}$. For $\mathrm{z}_{500}$, a principal component analysis (PCA) was performed, using the 120-year data sets (without spin-up period) for the $\mathrm{SH}$ and $\mathrm{NH}$ winter. The leading modes of variability (PC1) were interpreted as the modelled time series of $\mathrm{AAO}$ and $\mathrm{AO}$, and 8-year periods representing the respective oscillation phases were selected in COUP (Figures 3(a) and 3(b)) and REF (Figures 3(c) and 3(d)). A choice of high and low phases of the same length (8 years) from the reanalysed period 19581999 (Figures 3(e) and 3(f)), also based on a PCA at $\mathrm{Z}_{500}$, allows a reasonable comparison of ERA-40 data with the model results.

In addition, time filters were applied at each horizontal grid point in order to distinguish the long-term behaviour of the $\mathrm{z}_{500}$ field (low-pass filter) from superimposed short term fluctuations (band-pass filter). The band width of the lowpass filter (LP) was 10 to 90 days, and the band width of the band-pass filter (BP, Blackmon filter) was 2 to 6 days. 


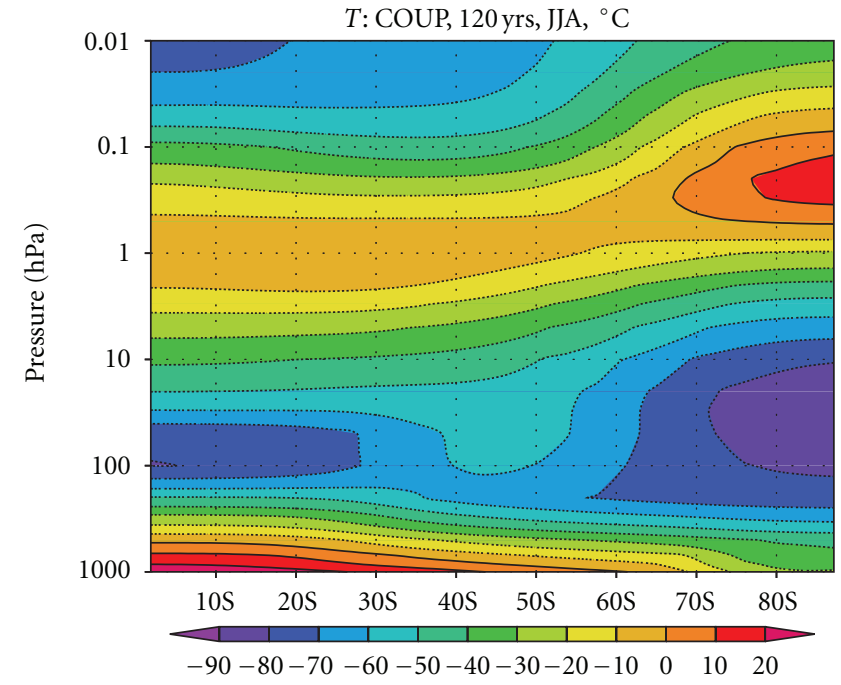

(a)

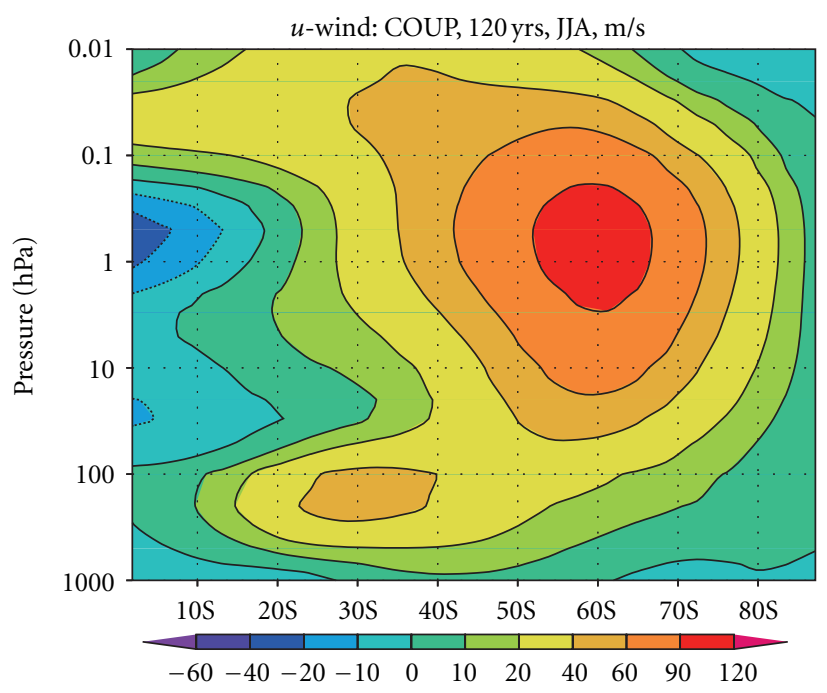

(c)

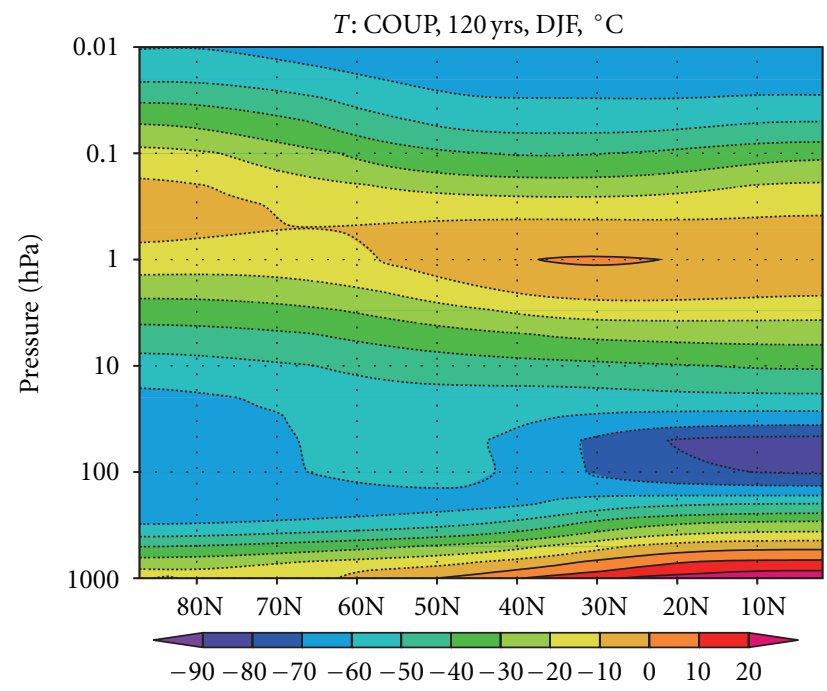

(b)

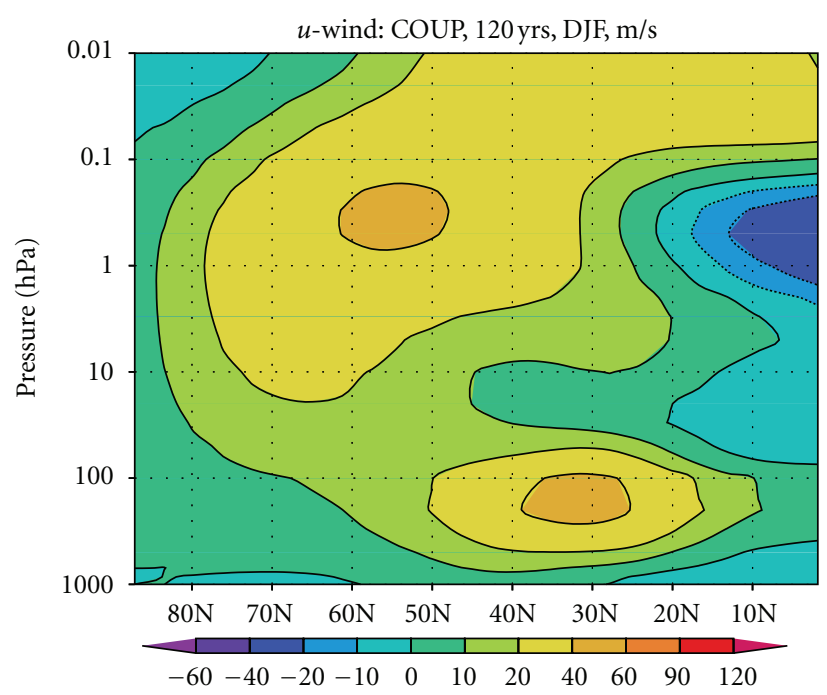

(d)

Figure 2: Model climatology. Simulation years from 31 to 150: zonal mean values within COUP. (a) Temperature $T$ in $\left({ }^{\circ} \mathrm{C}\right)$. JJA. $(\mathrm{b})$ Temperature $T$ in $\left({ }^{\circ} \mathrm{C}\right)$. DJF. (c) Zonal wind $u$ in (m/s). JJA. (d) Zonal wind $u$ in $(\mathrm{m} / \mathrm{s})$. DJF.

Using the filtered $\mathrm{z}_{500}$ data, discrete Fourier transformations (DFTs) for the SH winter were performed on each latitude slice. Based on this, the planetary-scale wavenumbers (WVNs) 1 to 3 from the LP-filtered field and the synoptic scale WVNs 4 to 10 from the BP-filtered field were compared between COUP and REF (Figure 4(b)). The squared wave amplitudes from the DFT are proportional to the respective wave energy at this WVN, while the logarithm of the wave energy (power) normalizes the spread of energy contents for different WVNs. The mean power of planetary and synoptic waves can thus be used as a measure of wave activity within COUP and REF.

For the discussion of wave dynamics, Eliassen-Palm fluxes (EP fluxes) were calculated for both AAO phases in the SH winter (JJA). In particular, the stationary component of the vertical EP flux is directly connected with planetary waves. Its divergence hints on the direction of the energy and momentum transfer between waves and tropospheric zonal mean flow, respectively, waves and strato-mesospheric polar vortex, but also on the direction of the meridional residual circulation (MRC). A negative sign of the EP flux divergence implies a tendency towards weakening of the mean flow, with improved conditions for wave propagation and/or a poleward MRC.

Significance tests for the results shown were based on monthly mean data. Using the hypothesis of unequal samples, a two-sided Student's $t$-test was applied. Within the figures, confidence areas are marked by white contour lines. An exception is Figure 4(b), where the $1 \sigma$ uncertainty intervals are given shaded. 


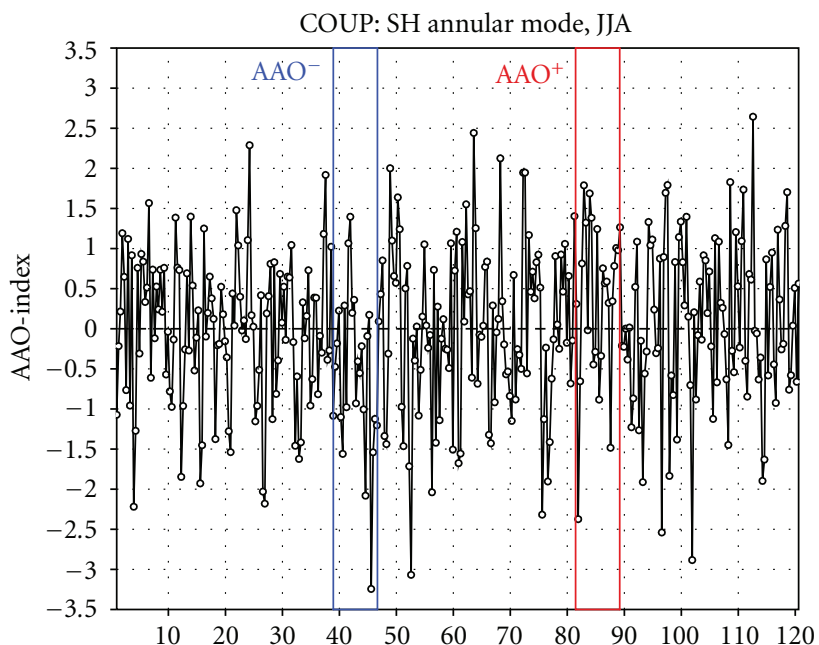

(a)

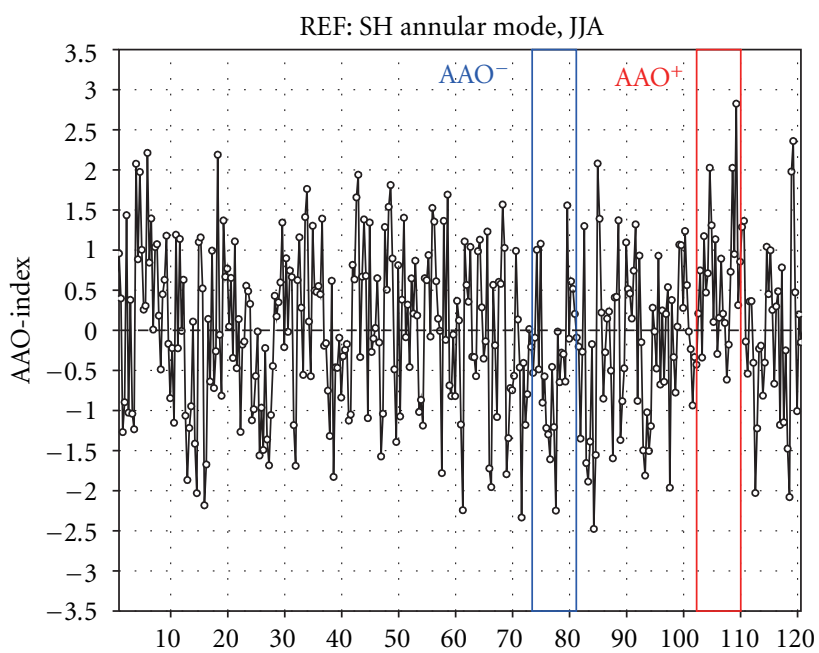

(c)

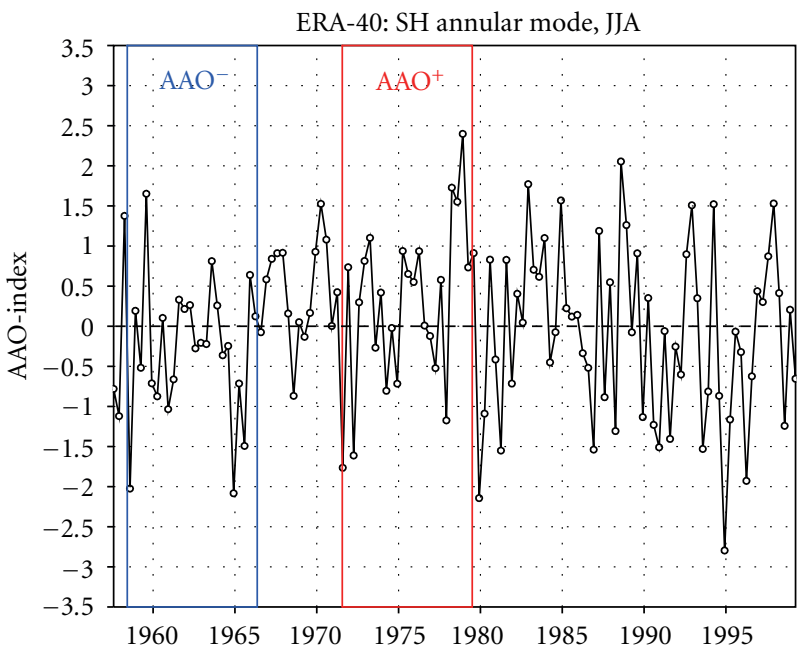

(e)

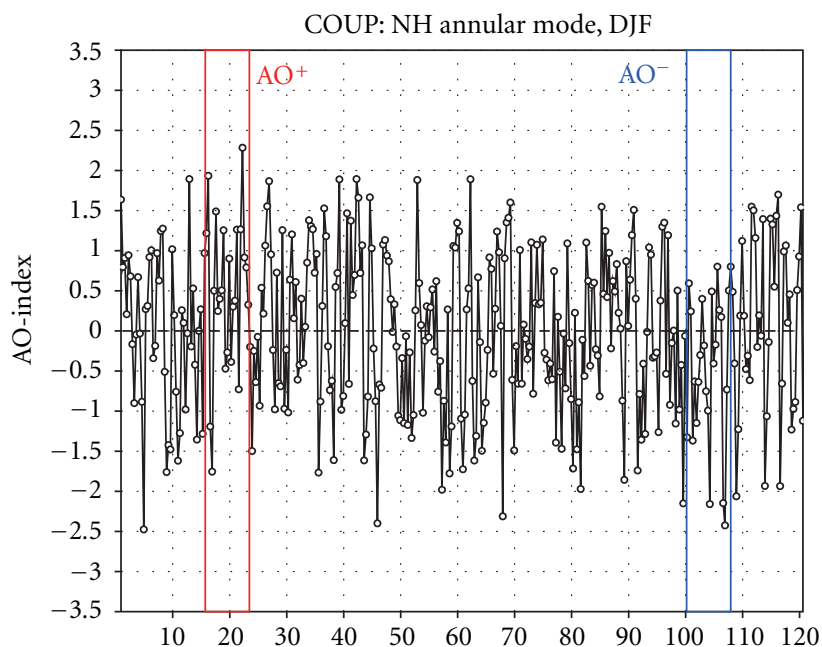

(b)

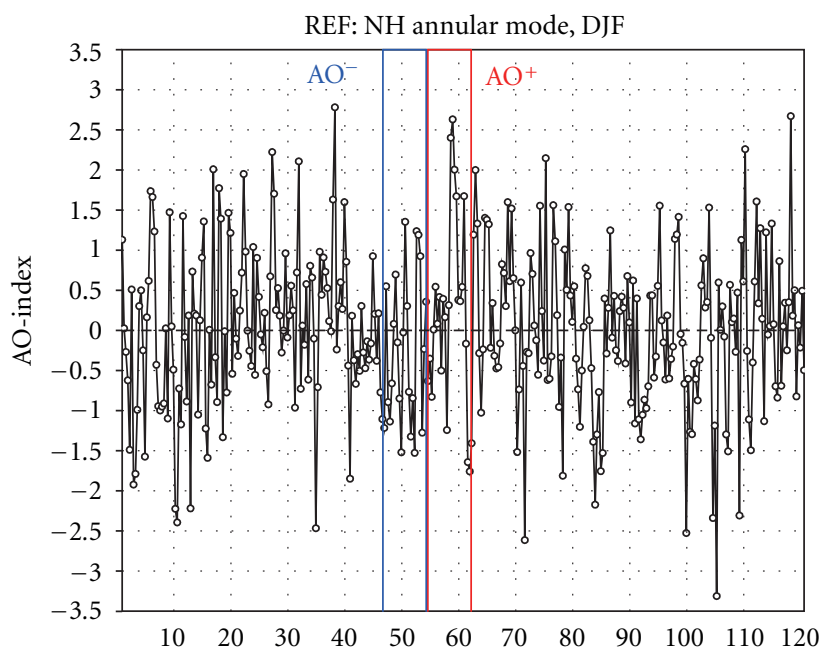

(d)

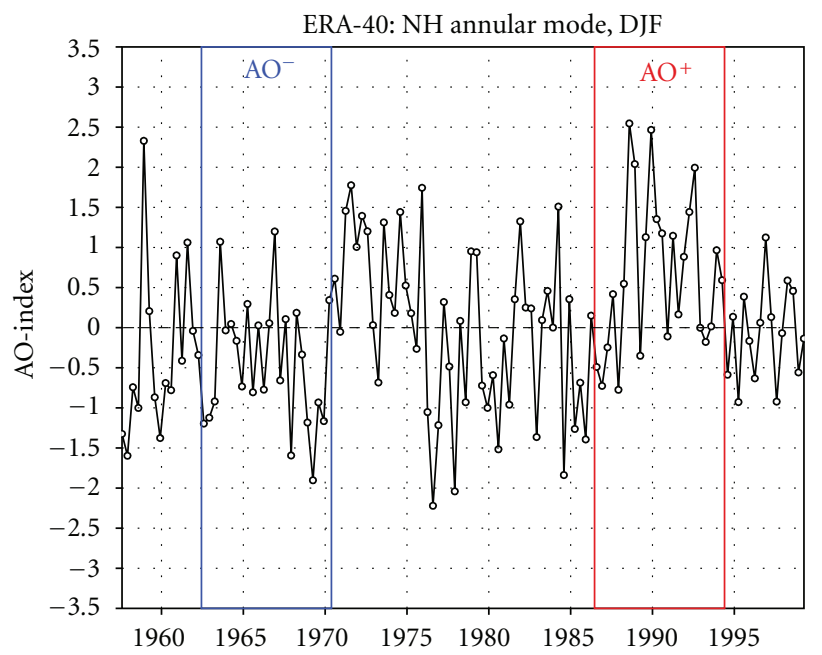

(f)

FIGURE 3: Annular modes. Principal component analysis (PCA) at $\mathrm{z}_{500}$. Leading mode of variability (PC1) in COUP, REF, and ERA-40 data. Name of the mode (AO/AAO) and explained variances are given in brackets. Left: JJA, SH. Right panels: DJF, NH. (a) COUP (AAO, 25.8\%). (b) $\operatorname{COUP}(\mathrm{AO}, 16.9 \%)$. (c) REF (AAO, 24.0\%). (d) REF (AO, 19.7\%). (e) ERA-40 (AAO, 22.5\%). (f) ERA-40 (AO, 18.4\%). For each dataset, sequences of 8 high- and low-index years (red/blue frames) were chosen as sample periods. 


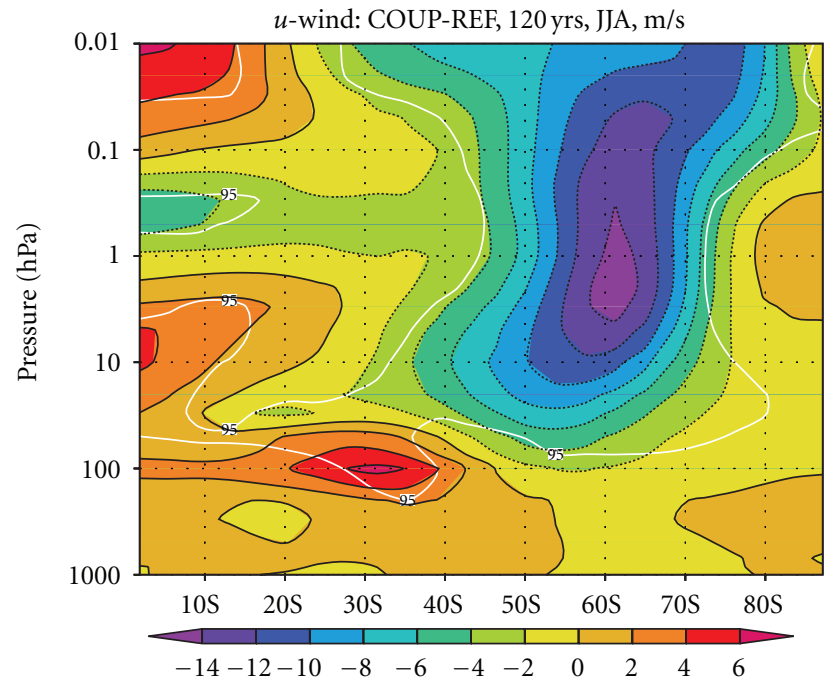

(a)

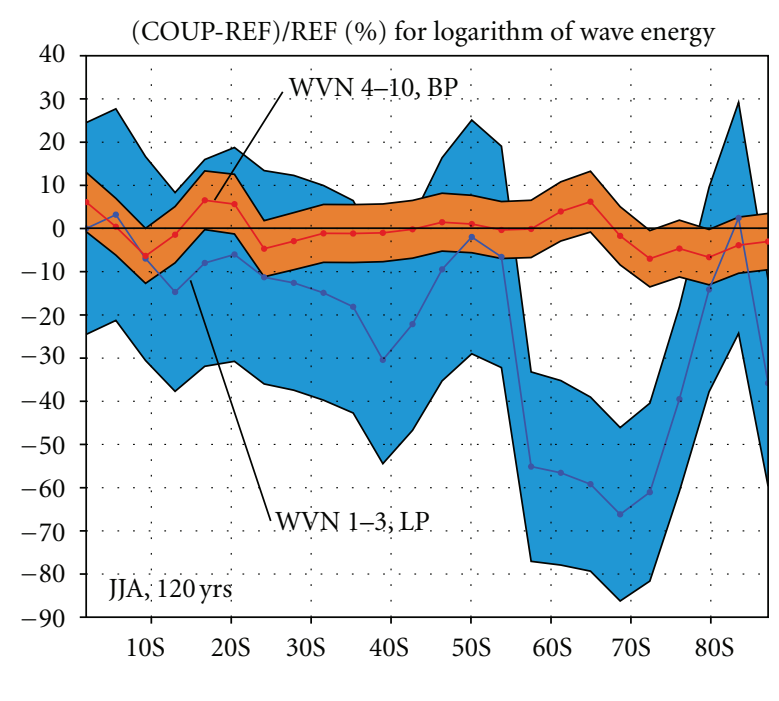

(b)

Figure 4: General evaluations. Simulation years from 31 to 150, JJA: (a) Zonal mean $u$ differences in (m/s) between COUP and REF. The white contour marks $95 \%$ confidence regions. (b) DFT of SH $z_{500}$ fields. Logarithm of wave energy, (COUP-REF)/REF in (\%). Blue: mean of LP (10-90 d) filtered WVNs 1-3, red: mean of BP (2-6 d)) filtered WVNs 4-10. $1 \sigma$ uncertainties are shaded.

\section{Results}

In order to discuss the SH winter (JJA) circulation within the two model runs, the first steps are the evaluation of zonal means and a discrete Fourier transformation (DFT) at $\mathrm{z}_{500}$. This level in the middle troposphere is most suitable for the analysis of tropospheric wave activity.

Figure 4(a) shows the zonal mean $u$ difference pattern between COUP and REF due to the interactive chemistrydynamics feedback. Since the first 30 years of each run were regarded as spin-up time, the results were averaged for the 120 model winters (DJF) 31 to 150 .

In mid- to high latitudes, the pattern shows a significant shift of COUP towards decreased strato-mesospheric zonal winds. It also hints on a weakened tropospheric zonal mean flow, while the subtropical jet within the troposphere is enhanced. In terms of the AAO atmospheric variability mode, this indicates a tendency towards $\mathrm{AAO}^{-}$for COUP compared to REF. Such a tropospheric weakening of the zonal mean flow and strengthening of the subtropical jet (i.e., anticorrelation of tropospheric zonal mean flow and subtropical jet) is for $\mathrm{AAO}^{-}(\mathrm{SH})$ and, more pronounced, $\mathrm{AO}^{-}(\mathrm{NH})$ also supported by ERA-40 reanalysis data (Figures 7(c) and 7(d)). Nevertheless, the modelled tropospheric effect is rather small and statistically not significant on a $95 \%$ confidence level (Figure $4($ a)), while the weakening of the strato-mesospheric polar vortex with 10 to $15 \mathrm{~m} / \mathrm{s}$ shows a much stronger, significant signal.

Since the differences for the zonal mean winds between COUP and REF show an AAO signature, also associated changes in the planetary- and synoptic-scale wave activity can be expected. Thus, a DFT of the filtered $z_{500}$ data was performed (see Section 2.3). The result is given in Figure 4(b). For the synoptic-scale waves, there is particularly in mid- to high latitudes no significant signal, while for the planetary-scale waves, a distinct and significant minimum in COUP relative to REF between around $55^{\circ} \mathrm{S}$ and $80^{\circ} \mathrm{S}$ can be noticed. So, overall COUP appears with reduced tropospheric planetary wave activity relative to REF, which seems to be in contradiction to the mean flow signal.

A weakened tropospheric mean flow in COUP should rather be connected with enhanced tropospheric wave activity, as energy of the mean flow can be redirected and cause additional wave formation. Finding the opposite result for the SH winter appears even more surprising, as for the same data sets (COUP compared to REF), the $\mathrm{NH}$ winter shows the expected dependency, that is, enhanced planetary waves in COUP (see Brand et al. [10]). Thus, a detailed analysis of the AAO (and AO) within the model simulations and a comparison with observations/reanalysis data (ERA40) are needed.

Based on a PCA of the $\mathrm{z}_{500}$ fields in REF and COUP, pronounced positive/negative AAO phases (JJA at $\mathrm{SH}$ ) and $\mathrm{AO}$ phases (DJF at $\mathrm{NH}$ ) were chosen in order to assess the variability due to Annular Modes within the simulations (see Section 2.3). Figure 5 shows the zonal mean differences of temperature $T$ and zonal wind $u$ between the positive and negative oscillation phases for COUP. Figure 6 shows the same for REF. In addition, comparable means from ERA-40 reanalysis data are presented in Figure 7 (see Section 2.3).

Comparing COUP (Figure 5) and REF (Figure 6), the most obvious finding is the different variability within the two simulations. With focus on mid- to high latitudes, the temperatures fluctuate between roughly $-2 \mathrm{~K}$ to $+2 \mathrm{~K}$ $(\mathrm{AAO}$, Figure $5(\mathrm{a}))$ and $-2 \mathrm{~K}$ to $+3 \mathrm{~K}(\mathrm{AO}$, Figure $5(\mathrm{~b}))$ in COUP, but $-4 \mathrm{~K}$ to $+5 \mathrm{~K}$ (AAO, Figure $6(\mathrm{a})$ ) and $-5 \mathrm{~K}$ to $+6 \mathrm{~K}$ (AO, Figure $6(\mathrm{~b})$ ) in REF. For the zonal wind, it is $-3 \mathrm{~m} / \mathrm{s}$ to $+3 \mathrm{~m} / \mathrm{s}$ (AAO, Figure $5(\mathrm{c})$ ) and $-2 \mathrm{~m} / \mathrm{s}$ to 


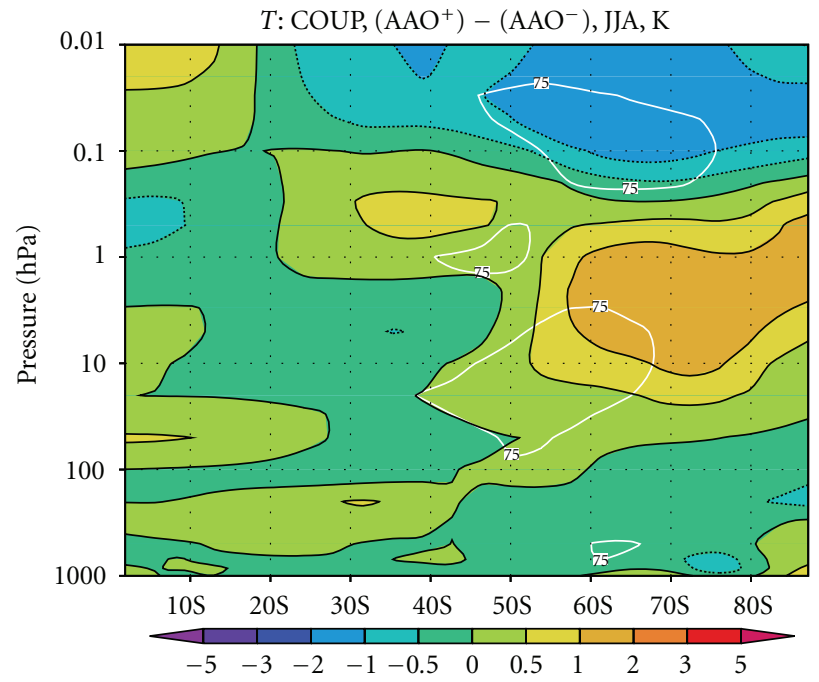

(a)

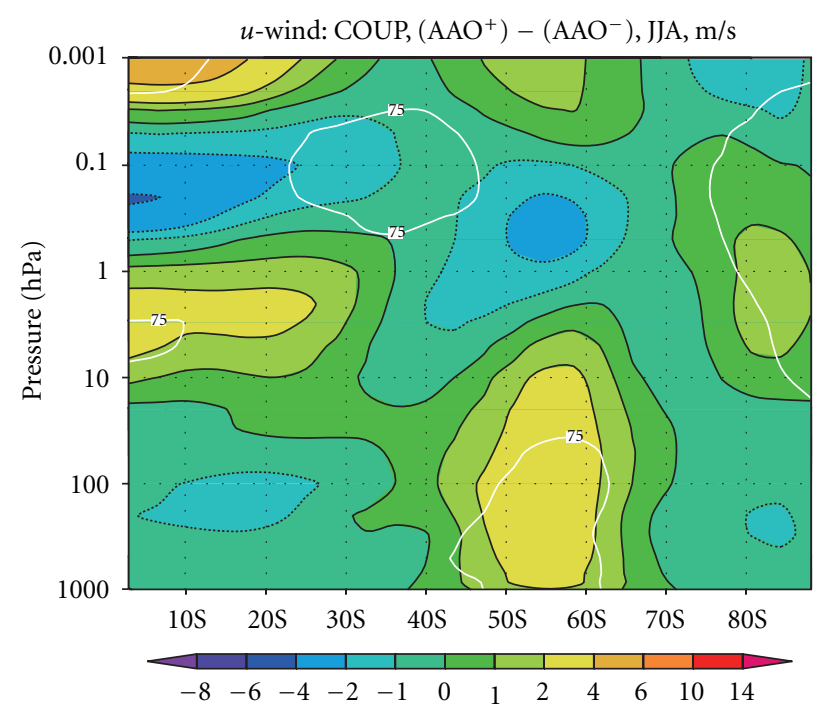

(c)

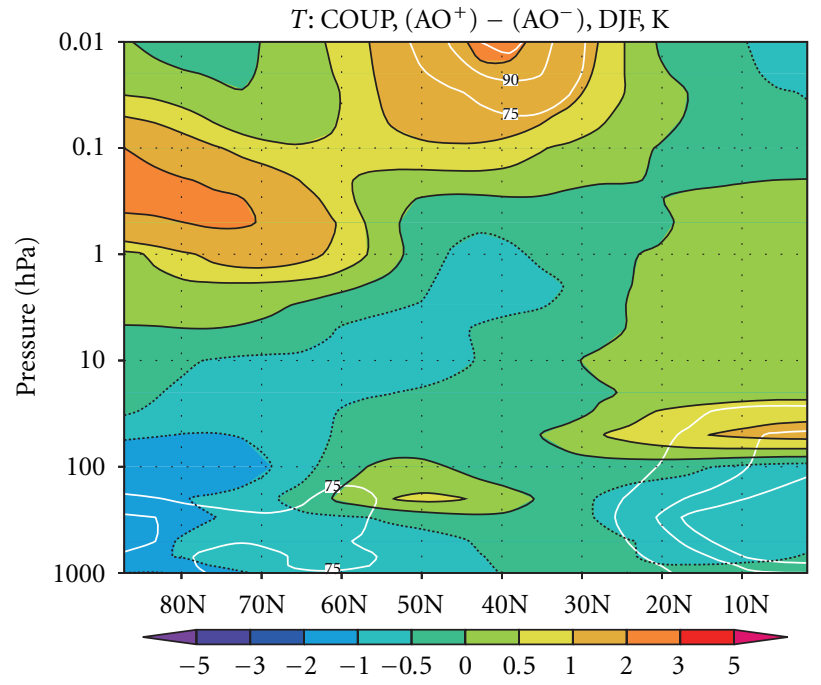

(b)

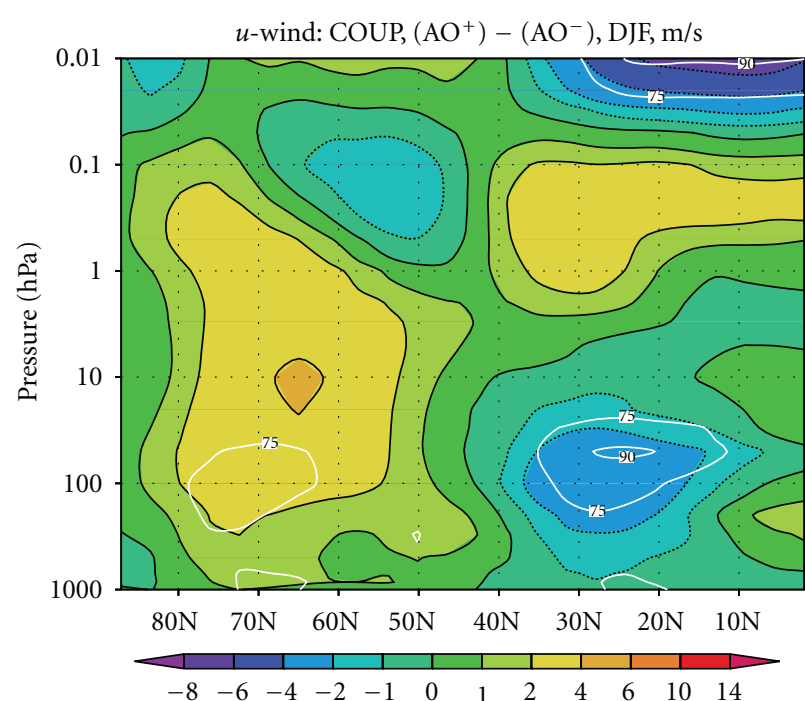

(d)

FIGURE 5: Annular modes (2). Model simulation: zonal mean differences within COUP between $\mathrm{AAO}^{+}$and $\mathrm{AAO}^{-}$, respectively, $\mathrm{AO}^{+}$and $\mathrm{AO}^{-}$. (a) Temperature $T$ in (K). JJA. (b) Temperature $T$ in (K). DJF. (c) Zonal wind $u$ in (m/s). JJA. (d) Zonal wind $u$ in (m/s). DJF. Confidence levels are given by white lines.

$+4 \mathrm{~m} / \mathrm{s}(A O$, Figure $5(\mathrm{~d}))$ in COUP, but $-8 \mathrm{~m} / \mathrm{s}$ to $+5 \mathrm{~m} / \mathrm{s}$ (AAO, Figure $6(\mathrm{c})$ ) and $0 \mathrm{~m} / \mathrm{s}$ to $+15 \mathrm{~m} / \mathrm{s}(\mathrm{AO}$, Figure $6(\mathrm{~d})$ ) in REF. This conforms to a factor of about 2 in all the above cases, where COUP yields the lower variability. At the same time, the AO variability is only slightly higher than the AAO variability, if concentrating on one of the simulations. Nevertheless, this is the absolute variability, while the relative variability (compared to the climatological values, see Figure 2) is clearly lower for the AAO due to the much stronger polar vortex in the $\mathrm{SH}$ winter.

As a result of the lower Annular Mode variability in COUP, also the significances of the signals go substantially down. Where REF shows significances on the 90 to $95 \%$ confidence levels for the main signals (Figure 6), the 75\% confidence level is hardly exceeded in COUP (Figure 5). Thus, it is not possible to discuss the variability within the interactive simulation standing alone. Only the comparison with the more reliable signals in REF as well as the variability patterns from ERA-40 (Figure 7) provides the chance to make use of these results from COUP. Consequently, we will argue some main aspects of the REF data first, before returning to COUP and examining similarities and differences within the two simulations.

For the tropospheric temperatures in REF (Figures 6(a) and 6(b)), there is a high latitudinal cooling within the positive phase of the oscillations in the respective winter hemisphere. This complies with the strengthening of the tropospheric midlatitudinal zonal mean flow 


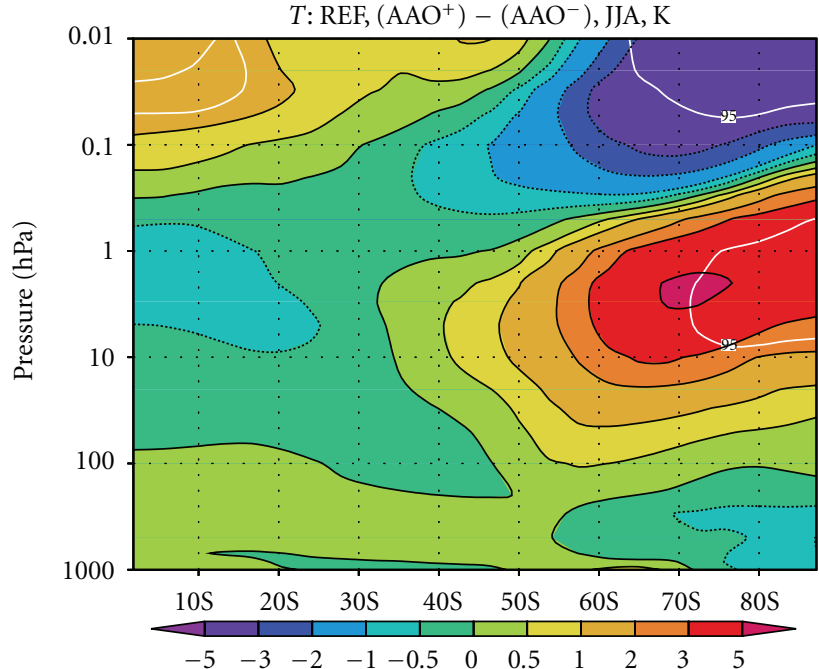

(a)

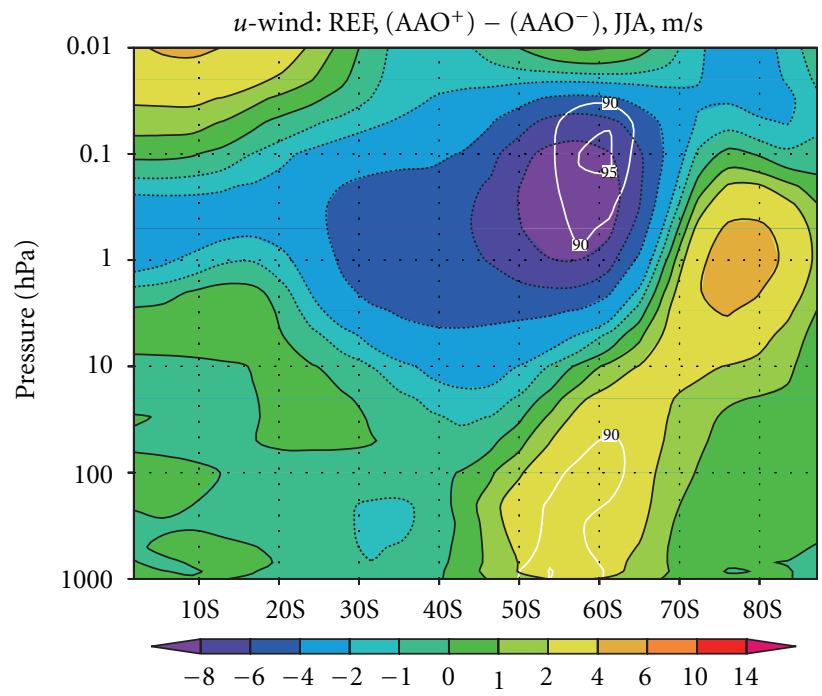

(c)

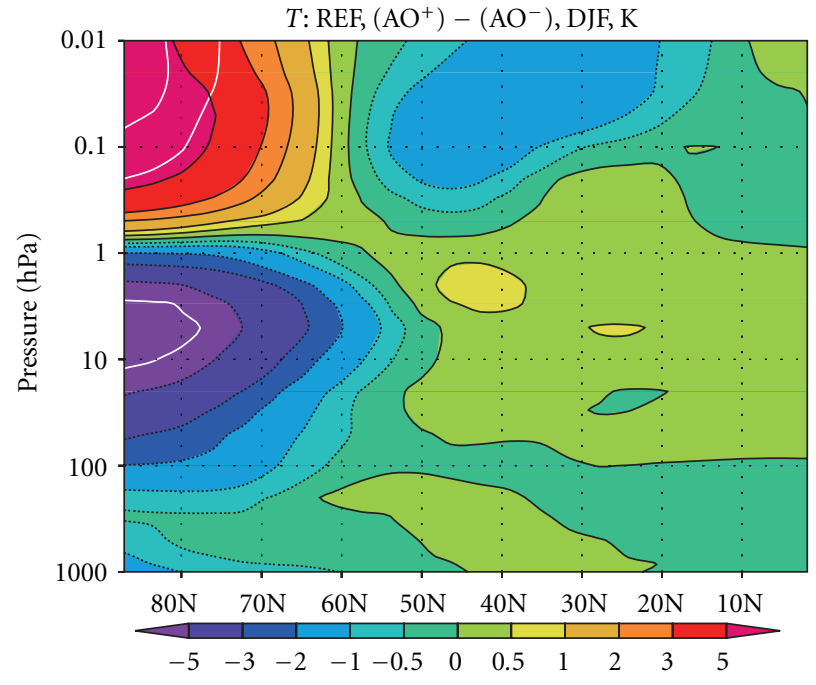

(b)

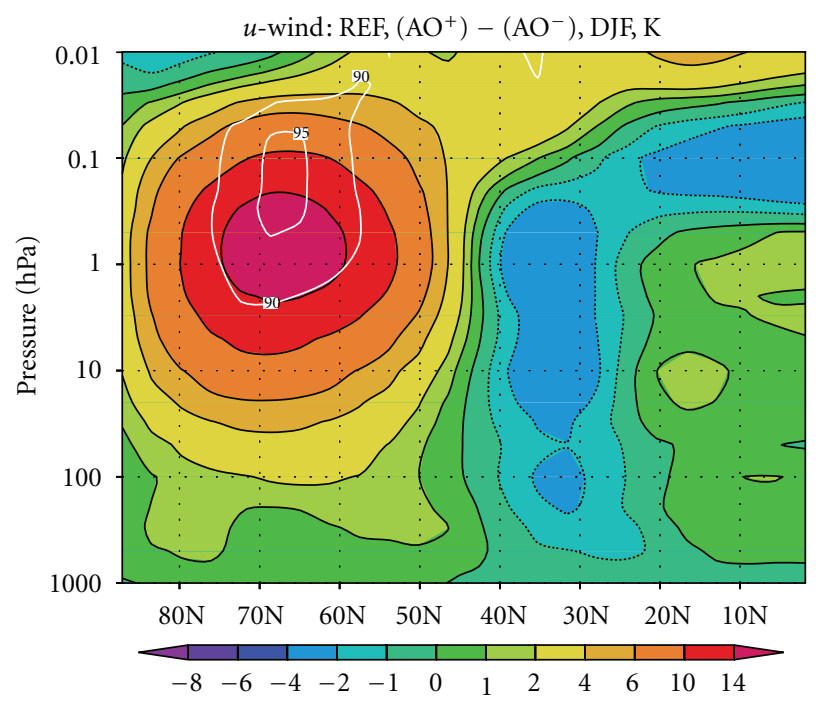

(d)

Figure 6: Annular modes (3). Model simulation: zonal mean differences within REF between $\mathrm{AAO}^{+}$and $\mathrm{AAO}^{-}$, respectively, $\mathrm{AO}^{+}$and $\mathrm{AO}^{-}$. (a) Temperature $T$ in (K). JJA. (b) Temperature $T$ in (K). DJF. (c) Zonal wind $u$ in (m/s). JJA. (d) Zonal wind $u$ in (m/s). DJF. Confidence levels are given by white lines.

(Figures 6(c) and 6(d)) and thus weakened meridional air mass exchange in this phase. Both effects are comparable to reanalysis data (ERA-40, Figure 7), but rather small, while the strato-mesospheric signals are considerably stronger. The vertical structure of the zonal wind differences shows a barotropic appearance throughout the troposphere, stratosphere, and lower mesosphere for the $\mathrm{NH}$ winter (Figure 6(d)), but yields an antisymmetric appearance between the troposphere/lower stratosphere and the upper stratosphere/mesosphere for the SH winter (Figure 6(c)).

The main signal in the $\mathrm{SH}$ winter midlatitudes in Figure 6(c) is the weakening of the strato-mesospheric polar vortex for $\mathrm{AAO}^{+}$, simultaneously to the strengthening of the zonal mean flow in the troposphere. A rather baroclinic vertical structure for the $\mathrm{SH}$ winter $\mathrm{AAO}$, contrary to a barotropic $\mathrm{NH}$ winter $\mathrm{AO}$ signal, is hinted also in ERA-40 (Figures $7(\mathrm{c})$ and $7(\mathrm{~d})$ ), although this data set ends at $1 \mathrm{hPa}$, and numerical edge effects might be important at the upper levels. However, within the model results, the SH winter antisymmetric AAO structure (Figure 6(c)) is significant on a confidence level of $90 \%$, as well as the opposing barotropic $\mathrm{AO}$ structure in the NH winter (Figure 6(d)). The same holds for the related strato-mesospheric $T$ differences (Figures 6(a) and 6(b)), having the same pattern and magnitude, but opposite signs of the temperature variations. In particular within the polar vortex, there is a stratospheric cooling under $\mathrm{AO}^{+}$conditions in the $\mathrm{NH}$ winter (Figure 6(b)), while the $\mathrm{SH}$ winter shows a warming for $\mathrm{AAO}^{+}$(Figure 6(a)).

Within COUP, apart from the lower Annular Mode variability and significance of the signals (see above), the $u$ 


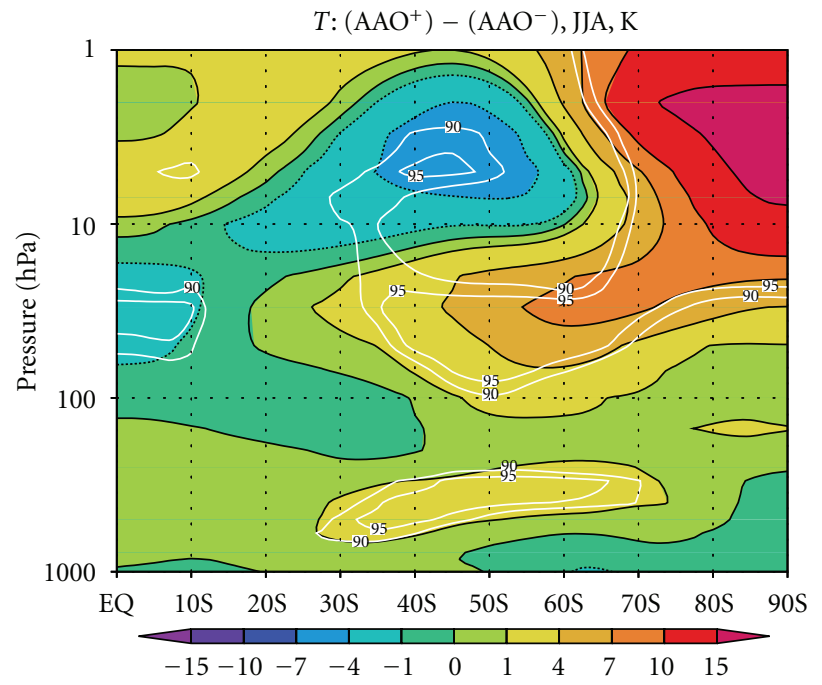

(a)

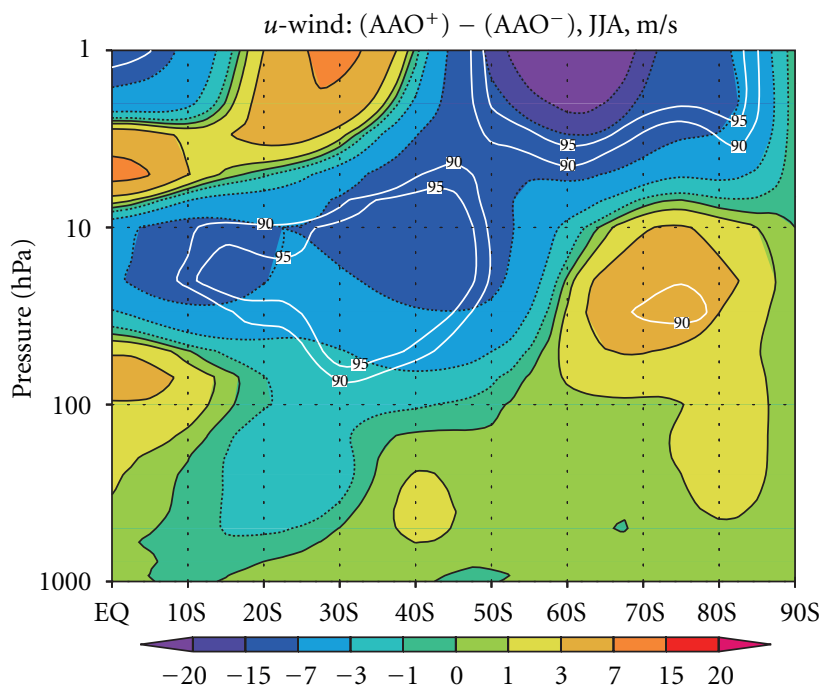

(c)

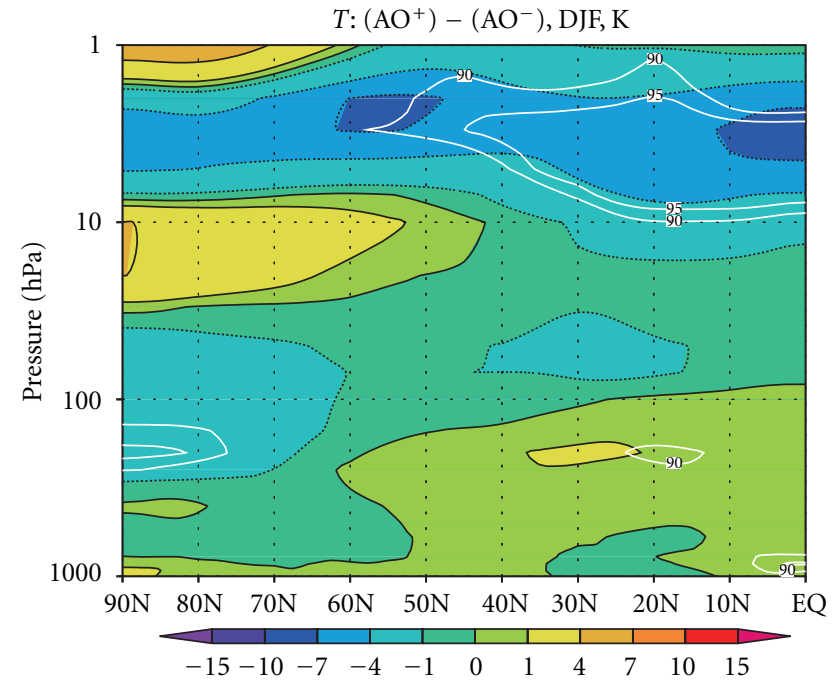

(b)

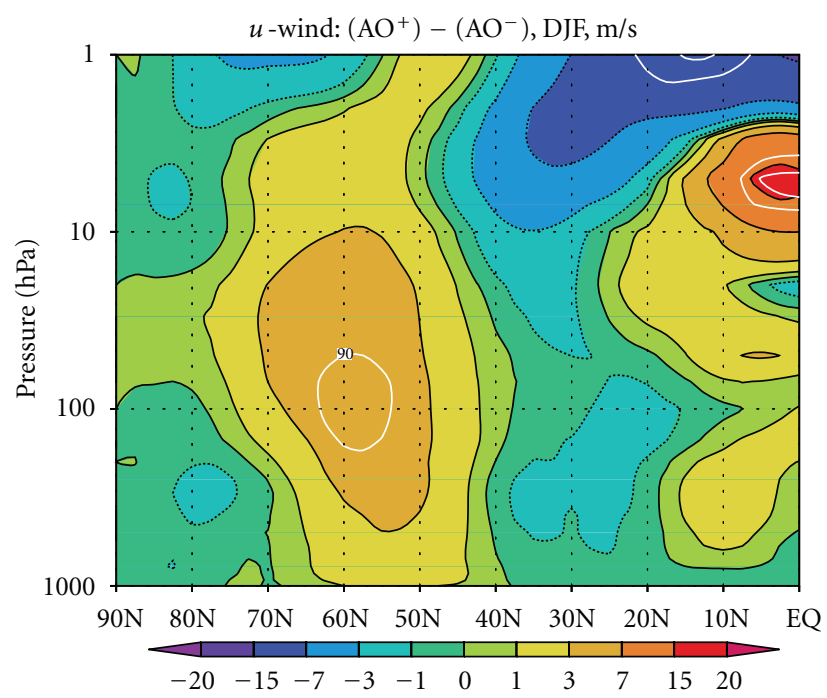

(d)

Figure 7: Annular modes (4). ERA-40 reanalysis data: zonal mean differences between $\mathrm{AAO}^{+}$and $\mathrm{AAO}^{-}$, respectively, $\mathrm{AO}^{+}$and $\mathrm{AO}^{-}$. (a) Temperature $T$ in (K). JJA. (b) Temperature $T$ in (K). DJF. (c) Zonal wind $u$ in (m/s). JJA. (d) Zonal wind $u$ in (m/s). DJF. Confidence levels are given by white lines.

and $T$ zonal mean patterns look strikingly similar to the ones from REF for the SH winter (Figures 5(a), 5(c), 6(a) and 6(c)), while some differences appear for the $\mathrm{NH}$ winter (Figures 5(b), 5(d), 6(b), and 6(d)). Especially south of about $60^{\circ} \mathrm{N}$ and above $10 \mathrm{hPa}$, the $\mathrm{AO}$ signal seems conversed, but without high significances $(>90 \%)$ in both simulations. Thus, taking into account the focus of this paper onto the $\mathrm{SH}$ winter circulation, this feature seems not to be relevant for the further discussion here. Below $10 \mathrm{hPa}$, as well as north of $60^{\circ} \mathrm{N}$, the zonal mean $\mathrm{NH}$ winter patterns from COUP and REF are rather similar to each other (Figures $5(\mathrm{~b}), 5(\mathrm{~d}), 6(\mathrm{~b})$, and $6(\mathrm{~d}))$, and the change of the planetary wave activity between the simulations hints on enhanced planetary waves in COUP (cp. above and see Brand et al. [10]).
For both simulations, the antisymmetric vertical structure of the modelled AAO, with strengthening/weakening of the tropospheric zonal mean flow and weakening/strengthening of the strato-mesospheric polar vortex for $\mathrm{AAO}^{+}$/ $\mathrm{AAO}^{-}$, appears specific for the $\mathrm{SH}$ (Figures 5(c) and 6(c)). At the same time, the SH winter COUP-REF pattern in Figure 4(a) is broadly barotropic, similar to the $\mathrm{NH}$ winter COUP-REF pattern (not shown here) and the modelled AO in Figures 5(d) and 6(d). Thus, although the SH winter zonal mean signal between the simulations (Figure $4(\mathrm{a})$ ) is $\mathrm{AAO}^{-}$ like within the troposphere, it appears $\mathrm{AAO}^{+}$like within the strato-mesosphere, if compared to the AAO zonal mean signal in Figures 5(c) and 6(c).

Aiming to further analyse the planetary wave activity, an evaluation of Eliassen-Palm (EP) fluxes was done for 


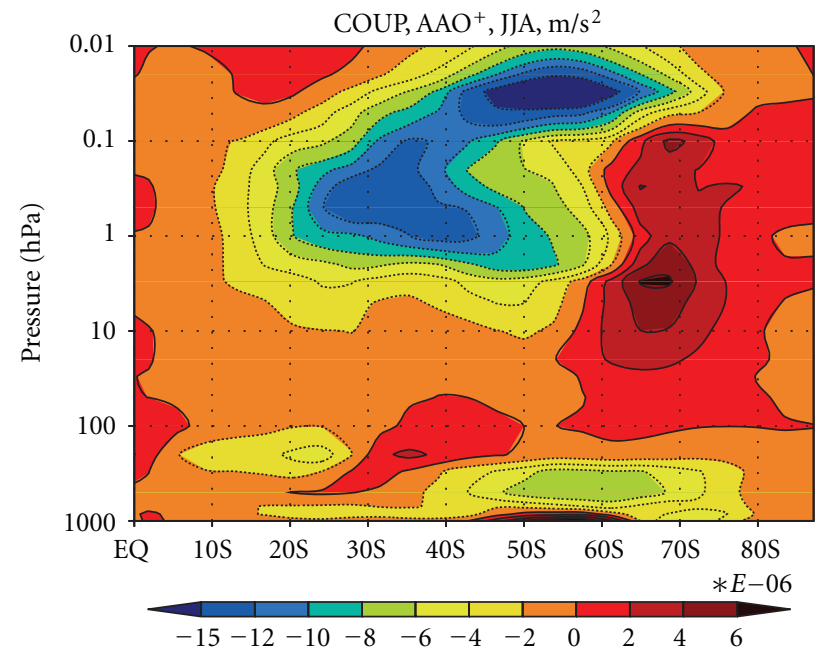

(a)

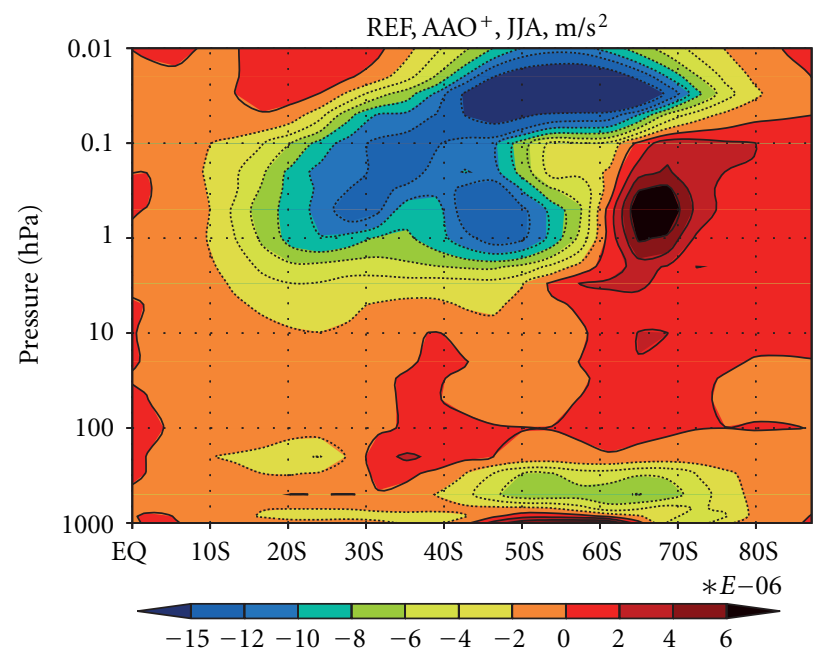

(c)

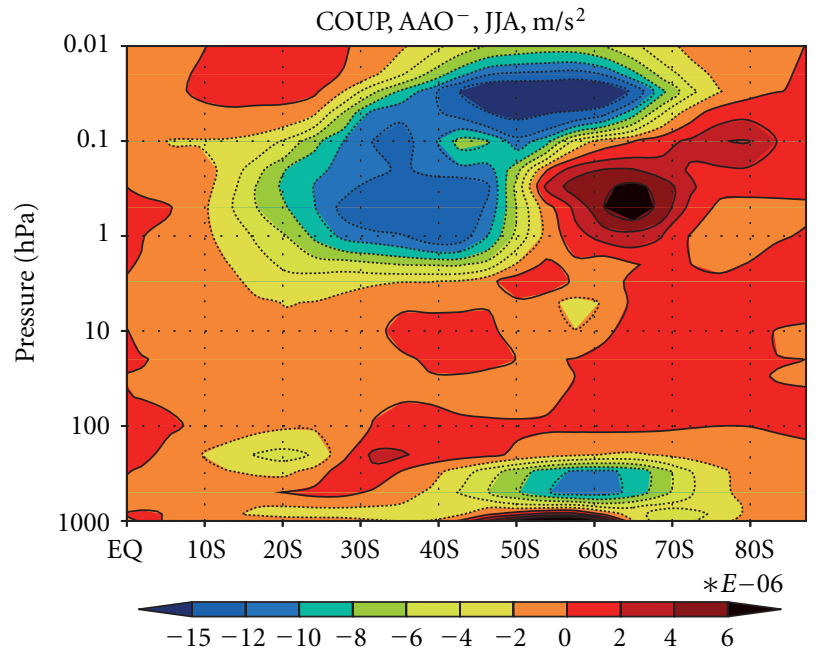

(b)

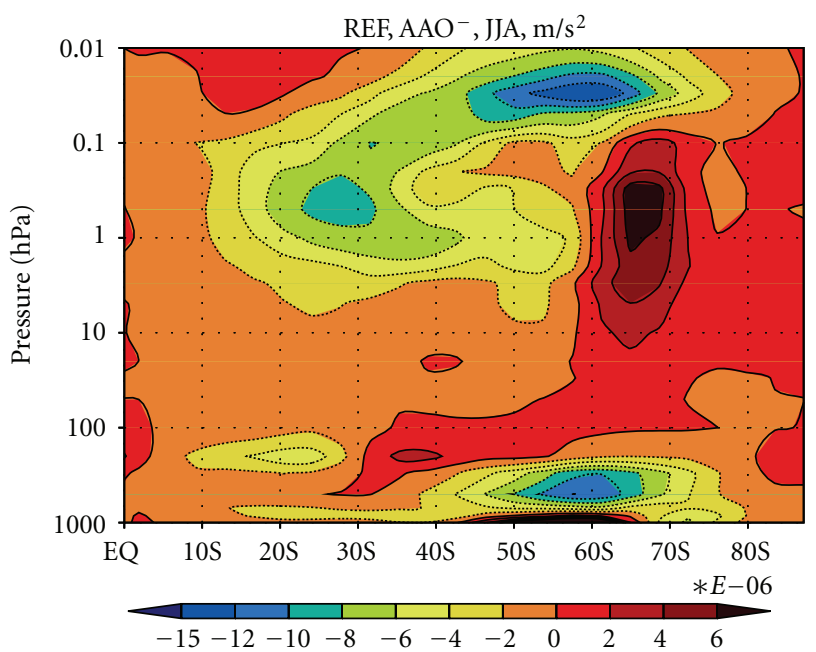

(d)

FIGURE 8: EP fluxes. Zonal means for the stationary component of the vertical EP fluxes. JJA results for $\mathrm{AAO}^{+}$and $\mathrm{AAO}^{-}$within $\mathrm{COUP}$ and REF. (a) Flux divergence for COUP $/ \mathrm{AAO}^{+}$in $\left(\mathrm{m} / \mathrm{s}^{2}\right)$. (b) Divergence for COUP $/ \mathrm{AAO}^{-}$in $\left(\mathrm{m} / \mathrm{s}^{2}\right)$. (c) Divergence for REF/AAO ${ }^{+}$in $\left(\mathrm{m} / \mathrm{s}^{2}\right)$. (d) Divergence for $\mathrm{REF} / \mathrm{AAO}^{-}$in $\left(\mathrm{m} / \mathrm{s}^{2}\right)$.

the $\mathrm{SH}$ winter $\mathrm{z}_{500}$ data (see Section 2.3). The divergence of the vertical stationary EP fluxes is given in Figure 8 for COUP (Figures 8(a) and 8(b)) and REF (Figures 8(c) and 8(d)). Thereby, the divergence illustrates energy and momentum transfer between waves and mean flow (e.g., tropospheric mean flow or stratospheric polar vortex) as well as changes of meridional air mass transport (e.g., meridional residual circulation, MRC) induced by fluctuations of the mean flow. The stationary flux component was chosen here, since it is connected with planetary waves.

Generally, there are three main features in Figures 8(a)8(d): a region with negative values within the troposphere around $60^{\circ} \mathrm{S}$ (R1), a region with positive values within the strato-mesosphere inside the polar vortex (south of $60^{\circ} \mathrm{S}$ and with varying heights, R2), and another region with negative values within the strato-mesosphere outside the polar vortex (north of $60^{\circ} \mathrm{S}$ and above $1 \mathrm{hPa}, \mathrm{R} 3$ ).
The tropospheric signal R1 indicates a weakening tendency of the tropospheric zonal mean flow, which is connected with planetary wave formation. Since the $\mathrm{AAO}^{-}$ phase of the Annular Mode is defined by a reduced tropospheric zonal mean flow compared to $\mathrm{AAO}^{+}$, it is natural to see a stronger R1 signal in this phase within both simulations (Figures $8(\mathrm{~b})$ and $8(\mathrm{~d})$ ), that is, improved conditions for wave stimulation due to energy released from the mean flow. Regarding the two strato-mesospheric signals, R2 marks the dissipation of the ascending planetary waves at the stratomesospheric polar vortex, while R3 denotes the poleward MRC above and around the upper part of the polar vortex.

Between COUP and REF, apparently the zonal mean patterns of the EP flux divergence are switched. $\mathrm{AAO}^{+}$ within COUP corresponds rather to $\mathrm{AAO}^{-}$within $\mathrm{REF}$ (Figures $8(\mathrm{a})$ and $8(\mathrm{~d})$ ), and $\mathrm{AAO}^{-}$within COUP to $\mathrm{AAO}^{+}$ within REF (Figures $8(\mathrm{~b})$ and $8(\mathrm{c})$ ). In addition, the strength 
of the R3 signal varies clearly for the reference simulation (Figures $8(\mathrm{c})$ and $8(\mathrm{~d})$ ), but only slightly for the interactive simulation (Figures $8(\mathrm{a})$ and $8(\mathrm{~b})$ ). Thus, the introduction of the interactive ozone chemistry in COUP shows a major effect here and alters the modelled planetary wave dynamics fundamentally. Thereby, the situation within COUP appears more reasonable: First, the AAO signal is relatively small compared to the strength of the strato-mesospheric polar vortex, and thus there should not be a strong impact on the MRC. Second, the R2 signal behaves in better accordance with the antisymmetric AAO zonal wind structure (Figures 5(c), 6(c) and 7(c)). Due to this, the strato-mesospheric polar vortex is weakened for $\mathrm{AAO}^{+}$, contrary to $\mathrm{AAO}^{-}$enabling the dissipation of upward propagating planetary waves also in the zonal wind maximum for this phase (Figures 8(a) and 8(b), cp. Figure 2(c)).

However, with respect to the reduced tropospheric planetary wave activity in COUP relative to REF (Figure 4(b)), the tropospheric situation is crucial. As discussed above, it hints on enhanced planetary wave activity for $\mathrm{AAO}^{-}$and thus declined activity for $\mathrm{AAO}^{+}$. Consequently, the wave formation within COUP also shows a more $\mathrm{AAO}^{+}$like signature compared to REF. Since the zonal mean SH winter signal between the simulations (Figure 4(a)) is $\mathrm{AAO}^{+}$like only within the strato-mesosphere, but $\mathrm{AAO}^{-}$like within the troposphere (see above), this suggests a mainly stratomesospheric control of the planetary wave dynamics.

\section{Summary and Conclusions}

Assuming a substantial impact of a more realistic stratospheric representation on the atmospheric circulation, as for the NH suggested by Shindell et al. [21] and underlined, for example, by the findings of Sigmond et al. [22] who analysed the tropospheric $\mathrm{AO}$ response to doubled $\mathrm{CO}_{2}$, interactive stratospheric chemistry effects might be even more crucial for the SH circulation. Thus, tropo- and strato-mesospheric signals within ECHO-GiSP results for the $\mathrm{SH}$ winter have been analysed. In the troposphere, the weakening of the midlatitudinal zonal mean flow between COUP and REF indicates a tendency of the coupled simulation towards a negative AAO. While this is comparable with the $\mathrm{NH}$ winter, the associated planetary wave activity, evaluated by a DFT of the $z_{500}$ data, behaves as opposed. Instead of being enhanced for COUP (enhanced planetary wave disturbances for weaker zonal mean flow), it significantly weakens.

Zonal mean temperature and zonal wind differences between $\mathrm{AAO}^{+}$and $\mathrm{AAO}^{-}$also demonstrate an opposing behaviour compared with the respective $\mathrm{NH}$ winter signal, mainly within the strato-mesosphere. There is an anticorrelation between the tropospheric and strato-mesospheric AAO signature, connecting a weak tropospheric flow with a strong polar vortex and vice versa. This antisymmetric pattern might be part of a negative feedback loop for the SH circulation: due to the vertical antisymmetry, a weakening of the strato-mesospheric polar vortex induces a strengthening of the tropospheric midlatitudinal zonal mean flow and a tendency towards reduced wave formation in the troposphere, leading to a weaker tropo-stratosphere coupling and thus stabilizing the polar vortex.

In a modelling context, it is an important question, to what extent it is necessary to resolve the strato-mesosphere as a whole, or whether it might also be sufficient only to have a high vertical model resolution in the lower stratosphere (see Gillett et al. [23]). The analysis of Eliassen-Palm fluxes for the SH winter planetary wave dynamics suggests the former, since the introduction of an interactive stratospheric ozone chemistry even converses the AAO signal in the zonal mean field of the EP flux divergence within our model simulations. Thereby, the situation in COUP appears to be more reasonable.

Given that the zonal wind difference between COUP and REF due to interactive stratospheric chemistry effects is rather barotropic, the middle atmospheric AAO response is not $\mathrm{AAO}^{-}$like as in the troposphere but shows an $\mathrm{AAO}^{+}$ tendency for COUP. Thus, it is possible to distinguish the direction of the mechanism controlling the planetary wave dynamics, depending on which AAO phase reveals a weakening of the tropospheric planetary wave activity. Because this holds for the $\mathrm{AAO}^{+}$phase, the control comes from the middle atmosphere. For both hemispheres, for example, Haynes et al. [9] also suggested a stratospheric downward control mechanism in winter due to the adjustment of the meridional residual circulation to planetary wave forcing.

To sum up, our model results show a strato-mesospheric control for the planetary wave dynamics in the $\mathrm{SH}$, and an overall zonal mean antisymmetric AAO signal between troposphere and strato-mesosphere. In comparison with the $\mathrm{NH}$ winter circulation, where the AO signal is rather barotropic throughout the tropo- and stratosphere, the baroclinic structure seems to be a specific $\mathrm{SH}$ feature and eventually contributed to large natural AAO variations and trends around 1960 and afterwards, as found in reconstructions for the 20th century SH summer by Jones and Widmann [24].

A potential explanation of the $\mathrm{SH}$ vertical asymmetry might be the different transmission of atmospheric waves through the stratospheric jets in the $\mathrm{NH}$ and $\mathrm{SH}$, which was (for gravity waves) mentioned by Kanzawa [25] while discussing observational results showing much warmer upper stratospheric temperatures in the Antarctic winter compared to the Arctic winter. Sensitivity experiments to evaluate the specific role of gravity waves for the SH circulation can potentially help to clarify this. However, it appears interesting that a baroclinic, antisymmetric AAO vertical structure as found here in a model context can also be detected within observational data. Unfortunately, although there are, for example, NCEP (up to $10 \mathrm{hPa}$ ), ERA-40, or UKMO reanalysis data sets, the sparse data availability within the SH stratomesosphere, especially in winter, still introduces substantial uncertainties.

\section{References}

[1] D. W. J. Thompson and J. M. Wallace, "Annular modes in the extratropical circulation. Part I: month-to-month variability," Journal of Climate, vol. 13, no. 5, pp. 1000-1016, 2000. 
[2] J. R. Holton, "The influence of gravity wave breaking on the general circulation of the middle atmosphere," Journal of the Atmospheric Sciences, vol. 40, no. 10, pp. 2497-2507, 1983.

[3] J. G. Charney and P. G. Drazin, "Propagation of planetaryscale disturbances from the lower into the upper atmosphere," Journal of Geophysical Research, vol. 66, pp. 83-109, 1961.

[4] R. L. Miller, G. A. Schmidt, and D. T. Shindell, "Forced annular variations in the 20th century Intergovernmental Panel on Climate Change Fourth Assessment Report models," Journal of Geophysical Research D, vol. 111, no. 18, Article ID D18101, 2006.

[5] D. T. Shindell and G. A. Schmidt, "Southern Hemisphere climate response to ozone changes and greenhouse gas increases," Geophysical Research Letters, vol. 31, no. 18, Article ID L18209, 2004.

[6] J. Perlwitz, S. Pawson, R. L. Fogt, J. E. Nielsen, and W. D. Neff, "Impact of stratospheric ozone hole recovery on Antarctic climate," Geophysical Research Letters, vol. 35, no. 8, Article ID L08714, 2008

[7] S. W. Son, L. M. Polvani, D. W. Waugh et al., "The impact of stratospheric ozone recovery on the Southern Hemisphere westerly jet," Science, vol. 320, no. 5882, pp. 1486-1489, 2008.

[8] A. A. Scaife, J. R. Knight, G. K. Vallis, and C. K. Folland, "A stratospheric influence on the winter NAO and North Atlantic surface climate," Geophysical Research Letters, vol. 32, no. 18, Article ID L18715, 2005.

[9] P. H. Haynes, C. J. Marks, M. E. McIntyre, T. G. Shepherd, and K. P. Shine, "On the "downward control" of extratropical diabatic circulations by eddy-induced mean zonal forces," Journal of the Atmospheric Sciences, vol. 48, no. 4, pp. 651-678, 1991.

[10] S. Brand, K. Dethloff, and D. Handorf, "Tropospheric circulation sensitivity to an interactive stratospheric ozone," Geophysical Research Letters, vol. 35, no. 5, Article ID L05809, 2008.

[11] S. Legutke and R. Voss, "The Hamburg atmosphere-ocean coupled circulation model ECHO-G," Tech. Rep. 18, DKRZ, Hamburg, Germany, 1999.

[12] R. Sander, A. Kerkweg, P. Jöckel, and J. Lelieveld, “Technical note: the new comprehensive atmospheric chemistry module MECCA," Atmospheric Chemistry and Physics, vol. 5, no. 2, pp. 445-450, 2005.

[13] E. Manzini, N. A. McFarlane, and C. McLandress, "Impact of the Doppler spread parameterization on the simulation of the middle atmosphere circulation using the MA/ECHAM4 general circulation model," Journal of Geophysical Research D, vol. 102, no. 22, pp. 25751-25762, 1997.

[14] E. Manzini and N. A. McFarlane, "The effect of varying the source spectrum of a gravity wave parameterization in a middle atmosphere general circulation model," Journal of Geophysical Research D, vol. 103, no. 24, pp. 31523-31539, 1998.

[15] E. Roeckner, K. Arpe, L. Bengtsson et al., "The atmospheric general circulation model ECHAM4: model description and simulation of present-day climate," Tech. Rep. 218, MPI for Met, Hamburg, Germany, 1996.

[16] J. O. Wolff, E. Maier-Reimer, and S. Legutke, "The Hamburg ocean primitive equation model HOPE,” Tech. Rep. 13, DKRZ, Hamburg, Germany, 1997.

[17] P. J. Rasch and D. L. Williamson, "On shape-preserving interpolation and semi-Lagrangian transport," SIAM Journal on Scientific and Statistical Computing, vol. 11, pp. 656-687, 1990.
[18] J. J. Morcrette, "Radiation and cloud radiative properties in the European Centre for Medium Range Weather Forecasts forecasting system," Journal of Geophysical Research, vol. 96, no. 5, pp. 9121-9132, 1991.

[19] H. Huebener, U. Cubasch, U. Langematz et al., "Ensemble climate simulations using a fully coupled ocean-tropospherestratosphere general circulation model," Philosophical Transactions of the Royal Society A, vol. 365, no. 1857, pp. 2089-2101, 2007.

[20] W. Kouker, I. Langbein, T. Reddmann, and R. Ruhnke, "The Karlsruhe Simulation Model of the Middle Atmosphere (KASIMA), version 2," Wissenschaftliche Berichte FZKA 6278, FZ Karlsruhe, Germany, 1999.

[21] D. T. Shindell, D. Rind, N. Balachandran, J. Lean, and P. Lonergan, "Solar cycle variability, ozone, and climate," Science, vol. 284, no. 5412, pp. 305-308, 1999.

[22] M. Sigmond, J. F. Scinocca, and P. J. Kushner, "Impact of the stratosphere on tropospheric climate change," Geophysical Research Letters, vol. 35, no. 12, Article ID L12706, 2008.

[23] N. P. Gillett, M. R. Allen, and K. D. Williams, "Modelling the atmospheric response to doubled $\mathrm{CO}_{2}$ and depleted stratospheric ozone using a stratosphere-resolving coupled GCM," Quarterly Journal of the Royal Meteorological Society, vol. 129, no. 589, pp. 947-966, 2003.

[24] J. M. Jones and M. Widmann, "Early peak in Antarctic oscillation index," Nature, vol. 432, no. 7015, pp. 290-291, 2004.

[25] H. Kanzawa, "Warm stratopause in the Antarctic winter," Journal of the Atmospheric Sciences, vol. 46, no. 3, pp. 435-438, 1989. 

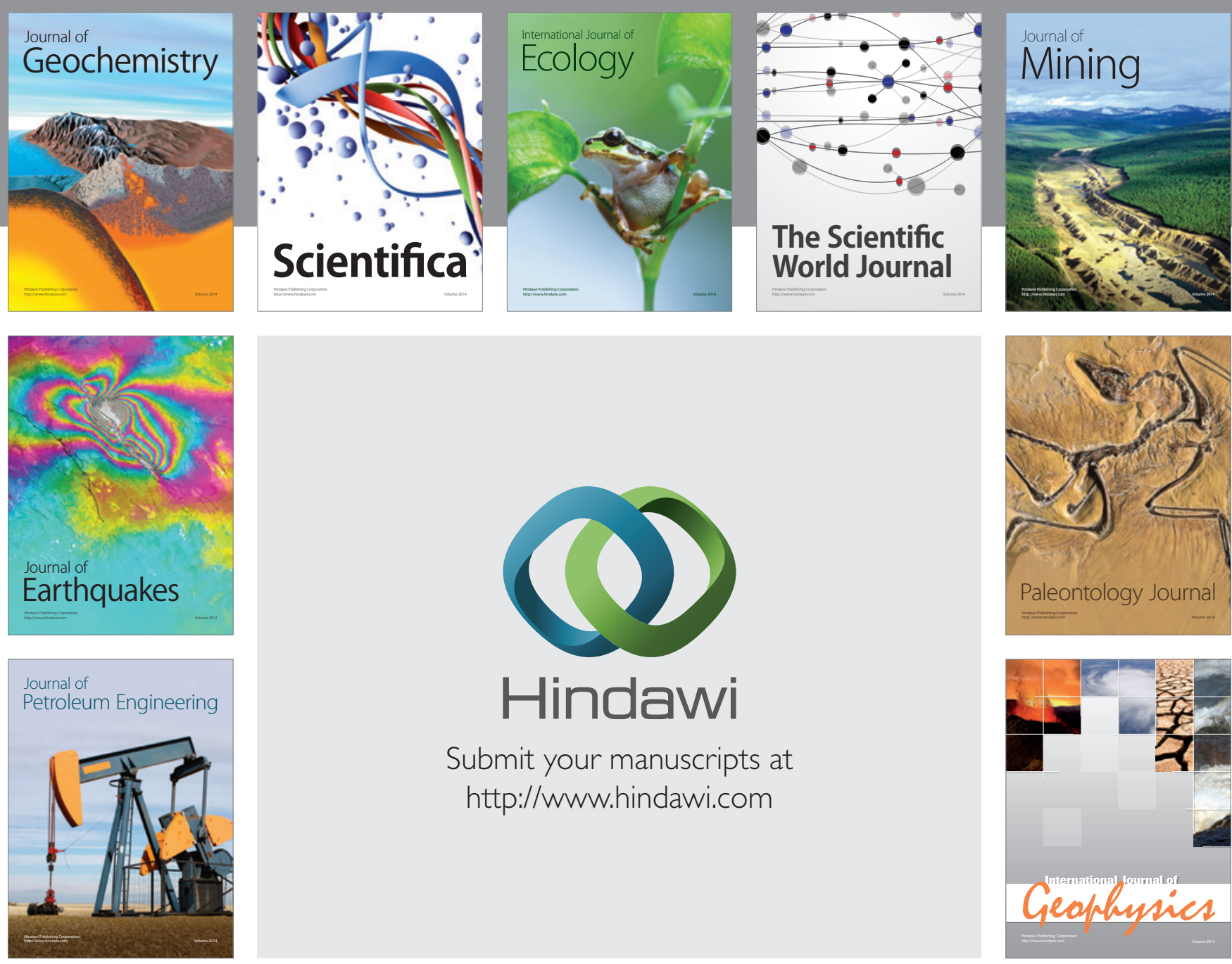

Submit your manuscripts at

http://www.hindawi.com
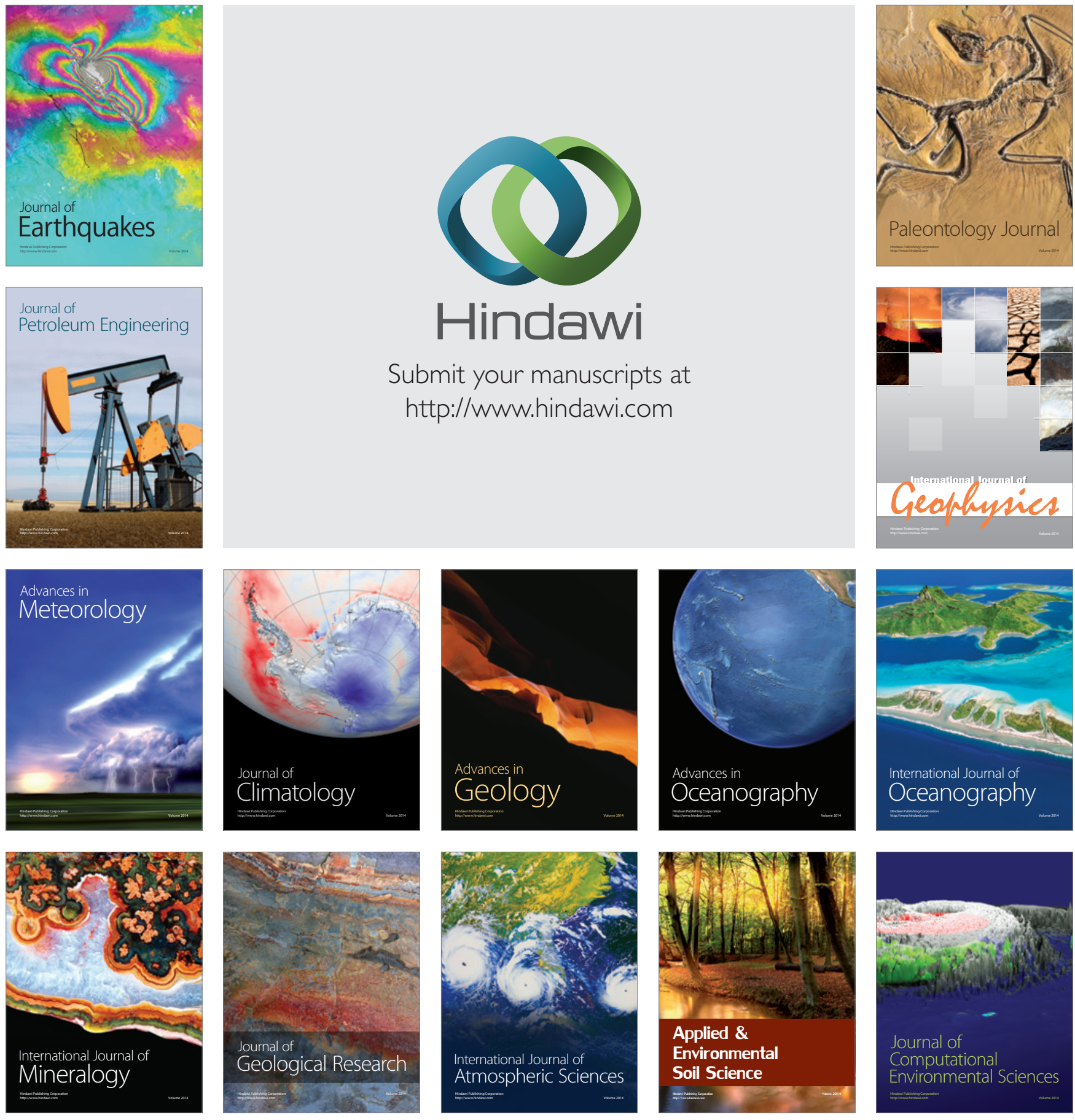